\title{
Blow-up and Critical Fujita Exponents in a Degenerate Parabolic Equation
}

\author{
Takefumi Igarashi \\ General Education Mathematics, College of Science and Technology, Nihon University, Chiba, 274-8501, Japan.
}

Received: May 04, 2016 / Accepted: June 02, 2016 / Published: July 25, 2016.

\begin{abstract}
In this paper, we consider the Cauchy problem of degenerate parabolic equation not in divergence form $u_{t}=u^{p} \Delta u+u^{q}, \quad p>1, \quad q>1$, and give the blow-up conditions and the critical Fujita exponents for the existence of global and non-global solutions to the Cauchy problem.
\end{abstract}

Key words: blow-up, global existence, critical exponent, degenerate parabolic equation.

\section{Introduction}

We study the Cauchy problem for the nonlinear diffusion equation not in divergence form

$$
\left\{\begin{array}{l}
u_{t}=u^{p} \Delta u+u^{q}, \quad x \in \mathbf{R}^{n}, t>0, \\
u(x, 0)=u_{0}(x), \quad x \in \mathbf{R}^{n},
\end{array}\right.
$$

where $n \geq 1, \quad p>1, \quad q>1$ and $0<u_{0}(x) \in$ $C\left(\mathbf{R}^{n}\right) \cap L^{\infty}\left(\mathbf{R}^{n}\right)$.

It is well known that problem (1) has a bounded positive continuous solution at least locally in time. (See [21, 22, 23].)

We define the blow-up time $T^{*}$ as

$$
T^{*}=\sup \{T>0 ;(1)
$$

possesses a solution in $\left.\mathrm{R}^{n} \times[0, T)\right\}$.

If $T^{*}=\infty$, the solution is global. On the other hand, if $T^{*}<\infty$, then the solution is not global in time in the sense that it blows up at $t=T^{*}$ such as

$$
\limsup _{t \rightarrow T^{*}}\|u(\cdot, t)\|_{L^{\infty}\left(\mathbf{R}^{n}\right)}=\infty \text {. }
$$

A lot of significant result on the critical exponents for nonlinear parabolic equations have been obtained during the past decades. Fujita [1] considered the

Corresponding author: Takefumi Igarashi, General Education Mathematics, College of Science and Technology, Nihon University, Chiba, 274-8501, Japan.
Cauchy problem:

$$
\left\{\begin{array}{c}
u_{t}=\Delta u+u^{p}, \quad x \in \mathbf{R}^{n}, t>0, \\
u(x, 0)=u_{0}(x), \quad x \in \mathbf{R}^{n} .
\end{array}\right.
$$

In [1], it is shown that (4) possesses the critical Fujita exponent $p^{*}=1+2 / n$ such that

- if $p \in(1, p *)$, then the solution $u(x, t)$ blows up in finite time for any $u_{0}(x)$;

- if $p \in(p *, \infty)$, then there are both global solutions and non-global solutions corresponding to small and large initial data, respectively.

Hayakawa [6], Kobayashi-Sirao-Tanaka [9] and Weissler [20] have known that $p=p^{*}=1+2 / n$ belongs to the blow-up case. In some situations, the size of initial data required by the global and non-global solutions can be determined via the so-called second critical exponent concerning the decay rates of initial data as $|x| \rightarrow \infty$. When $p>p^{*}=1+2 / n$, Lee-Ni [10] established the second critical exponent $a^{*}=2 /(p-1)$ for (4) with initial data $u_{0}(x)=\lambda \varphi(x)$, where $\lambda>0$ and $\varphi(x)$ is a bounded continuous function on $\mathbf{R}^{n}$, such that

- if $\quad \liminf |x|^{a} \varphi(x)>0 \quad$ for some

$$
|x| \rightarrow \infty
$$

$a \in\left(0, a^{*}\right)$ and any $\lambda>0$, then the solution 
$u(x, t)$ blows up in finite time;

- if $\limsup |x|^{a} \varphi(x)<\infty \quad$ for some $|x| \rightarrow \infty$

$a \in\left(a^{*}, n\right)$, and if $\lambda<\lambda_{0}$ for some $\lambda_{0}$, then the solution $u(x, t)$ is global.

Lee-Ni [10] proved that $a^{*}=2 /(p-1)$ belongs to the global case.

The degenerate case

$$
\left\{\begin{array}{l}
u_{t}=\Delta u^{m}+u^{p}, \quad x \in \mathbf{R}^{n}, t>0, \\
u(x, 0)=u_{0}(x), \quad x \in \mathbf{R}^{n},
\end{array}\right.
$$

with $\quad m>1$ and $\max (0,1-2 / n)<m<1 \quad$ were thoroughly studied with the critical Fujita exponent $p^{*}=m+2 / n \quad$ by Galaktionov-KurdyumovMikhailov-Samarskii [3], Mochizuki-Mukai [12] and Qi [16]. Furthermore, Galaktionov [2], Mochizuki-Mukai [12], Kawanago [8] and Mochizuki-Suzuki [13] have shown that $p=p^{*}=m+2 / n$ belongs to the blow-up case. When $\quad p>p^{*}=m+2 / n$

Mukai-Mochizuki-Huang [14] and Guo-Guo [5] obtained the second critical exponent $a^{*}=2 /(p-m)$ for $(5)$.

It is mentioned that the degenerate equation (5) can be changed to

$$
v_{t}=v^{\alpha} \Delta v+v^{\beta}
$$

under the transformation $u(x, t)=a v^{m}(b x, t)$, $a=m^{m /(p-1)}, \quad b=m^{(p-m) / 2(p-1)}$, with the special $\alpha=(m-1) / m<1$ and $\beta=(m+p-1) / m>1$. Obviously, the equations (5) and (6) are not equivalent to each other for general $\alpha$. Winkler [22] considered the Cauchy problem (1) for $p \geq 1$, and obtained the following results:

- For $1 \leq q<p+1$ (resp. $1 \leq q<3 / 2$ if $p=1$ ), all positive solutions of (1) are global and unbounded, provided that $u_{0}(x)$ decreases sufficiently fast in space.

- For $q=p+1$, all positive solutions of (1) blow up in finite time.

- For $q>p+1$, there are both global and non-global positive solutions.

Later, Li-Mu [11] and Yang-Zheng-Zhou [23] considered the Cauchy problem (1) for $p>1$, and obtained the following results when $q>p+1+2 / n$ :

(i) Let $n \geq 2$. Assume that $u_{0}(x)=\lambda \varphi(x)$, where $\lambda>0$ and $\varphi(x)$ is a bounded continuous function in $\mathbf{R}^{n}$, and that

$$
\liminf _{|x| \rightarrow \infty}|x|^{a} \varphi(x)>0 .
$$

If

$$
a \in\left(0, \frac{2}{q-p-1}\right)
$$

or

$$
a=\frac{2}{q-p-1} \text { with large } \lambda>0
$$

then the solution $u(x, t)$ blows up in finite time.

(ii) Let $n \geq 1$. Assume that $u_{0}(x)=\lambda \varphi(x)$, and that

$$
\limsup _{|x| \rightarrow \infty}|x|^{a} \varphi(x)<\infty .
$$

If

$$
a \in\left(\frac{2}{q-p-1}, n\right),
$$

then there exist $\lambda_{0}=\lambda_{0}(\varphi), C>0$ such that the solution $u(x, t)$ is global in time and satisfies

$$
\|u(x, t)\|_{L^{\infty}\left(\mathbf{R}^{n}\right)} \leq C t^{-a /(a p+2)}
$$

for all $t>0$ whenever $\lambda<\lambda_{0}$.

The conclusions (i)-(ii) show that the problem (1) admits the second critical exponent

$$
a^{*}=\frac{2}{q-p-1}
$$

with $n \geq 2$ and $q>p+1+2 / n$.

In this article, we will study the blow-up of solutions $u(x, t)$ to the Cauchy problem (1) when $p+1<q \leq p+1+2 / n$ or $0<a<2 /(q-p-1)$ with $n \geq 1$. 
Theorem Let $n \geq 1$. Suppose that one of the following two conditions holds;

(a) $p+1<q \leq p+1+\frac{2}{n}$.

(b) $\liminf _{|x| \rightarrow \infty}|x|^{a} u_{0}(x)>0$ with $0<a<\frac{2}{q-p-1}$.

Then the solution $u(x, t)$ of (1) blows up in finite time.

Comparing Theorem and the conclusions of $\mathrm{Li}-\mathrm{Mu}$ [11] and Yang-Zheng-Zhou [23], we see that (1) possesses the critical Fujita exponent

$$
q^{*}=p+1+\frac{2}{n}
$$

and the second critical exponent

$$
a^{*}=\frac{2}{q-p-1}
$$

with $n \geq 1$ and $q>p+1$, and may be summarized in the following table:

\begin{tabular}{|l|l|l|}
\hline & $p+1<q \leq q^{*}$ & $q>q^{*}$ \\
\hline$a<a^{*}$ & Blow - up & Blow - up \\
\hline$a=a^{*}$ & Blow - up & Blow - up for large data \\
\hline$a>a^{*}$ & Blow - up & $\begin{array}{l}\text { Global for small data } \\
\text { Blow - up for large data }\end{array}$ \\
\hline
\end{tabular}

The rest of this paper is organized as follows. In sections 2 and 3, we state the proof of Theorem for the conditions (a) and (b), respectively.

\section{Proof of Theorem (a)}

In this section, we shall prove the Theorem for the condition (a) by two case of $n \geq 2$ and $n=1$.

\subsection{Case I: $n \geq 2$}

We take the same strategy as in Li-Mu [11] and Igarashi-Umeda [7].

Let

$$
B_{r, m}=\left\{x \in \mathbf{R}^{n} ;\left|x-x_{m}\right|<r\left|x_{m}\right|\right\}
$$

for some constant $r>0$ and a sequence $\left\{x_{m}\right\}_{m=1}^{\infty}$ satisfying $0<\left|x_{m}\right|<\left|x_{m+1}\right|$ for any $m \in \mathbf{N}$ and $\lim _{m \rightarrow \infty}\left|x_{m}\right|=\infty$.

Remark The method using the sequence of balls $B_{r, m}$ in (16) was used in $[17,4,18,19]$ and the other papers.

Let $\lambda_{m}>0$ denote the principal eigenvalue of $-\Delta$ with Dirichlet problem in $B_{r, m}$, and let $\varphi_{m}(x)$ denote the corresponding eigenfunction, normalized by

$$
\int_{B_{r, m}} \varphi_{m}(x) d x=1
$$

We define

$$
F_{m}(t)=\frac{1}{p-1} \int_{B_{r, m}} u^{1-p}(x, t) \varphi_{m}(x) d x .
$$

Then we have

$$
\begin{aligned}
& F_{m}^{\prime}(t)=-\int_{B_{r, m}} \frac{u_{t}}{u^{p}} \varphi_{m}(x) d x \\
& =-\int_{B_{r, m}}\left(\Delta u+u^{q-p}\right) \varphi_{m}(x) d x .
\end{aligned}
$$

Integrating by parts, using the fact that $\varphi_{m}=0$ and $\partial \varphi_{m} / \partial v \leq 0$ on $\partial B_{r, m}$, where $v$ denote the outward unit normal vector to $B_{r, m}$ at $x \in \partial B_{r, m}$, and applying Green's formula, we obtain

$$
F_{m}^{\prime}(t) \leq \lambda_{m} \int_{B_{r, m}} u \varphi_{m}(x) d x-\int_{B_{r, m}} u^{q-p} \varphi_{m}(x) d x .
$$

Since $B_{r, m}$ is a $n$-dimensional ball of radius $r\left|x_{m}\right|$, it follows that $\lambda_{m}$ satisfies

$$
\lambda_{m} \leq \frac{c}{\left|x_{m}\right|^{2}}
$$

where $c>0$ depends only on the dimension $n$ and $r$. Thus, we have

$$
\begin{aligned}
F_{m}^{\prime}(t) & \leq \frac{c}{\left|x_{m}\right|^{2}} \int_{B_{r, m}} u \varphi_{m}(x) d x \\
& -\int_{B_{r, m}} u^{q-p} \varphi_{m}(x) d x
\end{aligned}
$$


Since $p>1, q-p>1$, and $\int_{B_{r, m}} \varphi_{m}(x) d x=1$, by Jensen' s and Hölder' s inequalities, we have

$$
\int_{B_{r, m}} u^{1-p} \varphi_{m}(x) d x \geq\left(\int_{B_{r, m}} u \varphi_{m}(x) d x\right)^{1-p}
$$

and

$$
\int_{B_{r, m}} u^{q-p} \varphi_{m}(x) d x \geq\left(\int_{B_{r, m}} u \varphi_{m}(x) d x\right)^{q-p} .
$$

It follows from (23)-(24) that

$$
\begin{gathered}
\int_{B_{r, m}} u^{q-p} \varphi_{m}(x) d x \\
\geq\left(\int_{B_{r, m}} u \varphi_{m}(x) d x\right)^{q-p-1} \int_{B_{r, m}} u \varphi_{m}(x) d x \\
\geq\left(\int_{B_{r, m}} u^{1-p} \varphi_{m}(x) d x\right)^{-\frac{q-p-1}{p-1}} \int_{B_{r, m}} u \varphi_{m}(x) d x \\
=\left[(p-1) F_{m}(t)\right]^{-(q-p-1) /(p-1)} \int_{B_{r, m}} u \varphi_{m}(x) d x .
\end{gathered}
$$

Thus, by (22)-(25), we obtain

$$
\begin{gathered}
F_{m}^{\prime}(t) \leq \frac{c}{\left|x_{m}\right|^{2}} \int_{B_{r, m}} u \varphi_{m}(x) d x \\
-\left[(p-1) F_{m}(t)\right]^{-(q-p-1) /(p-1)} \int_{B_{r, m}} u \varphi_{m}(x) d x .
\end{gathered}
$$

Here, if $F_{m}(t)$ satisfies

$$
F_{m}(t) \leq \frac{1}{p-1}\left(\frac{\left|x_{m}\right|^{2}}{2 c}\right)^{(p-1) /(q-p-1)}
$$

for all $t \in\left[0, T^{*}\right)$, then by (26), it follows that

$$
F_{m}^{\prime}(t) \leq-\frac{c}{\left|x_{m}\right|^{2}} \int_{B_{r, m}} u \varphi_{m}(x) d x .
$$

Hence, if (28) holds, then by (23)-(28), we have

$$
F_{m}^{\prime}(t) \leq-\frac{c}{\left|x_{m}\right|^{2}}(p-1)^{\frac{1}{1-p}} F_{m}^{\frac{1}{1-p}}(t)
$$

from which it follows that if $F_{m}(0)$ satisfies

$$
F_{m}(0) \leq \frac{1}{p-1}\left(\frac{\left|x_{m}\right|^{2}}{2 c}\right)^{\frac{p-1}{q-p-1}},
$$

then $F_{m}(t)$ decreases and

$$
F_{m}(t)<\frac{1}{p-1}\left(\frac{\left|x_{m}\right|^{2}}{2 c}\right)^{\frac{p-1}{q-p-1}} \text { for all } t \in\left[0, T^{*}\right]
$$

and an integration of (29) shows that

$$
F_{m}(t) \leq\left(F_{m}^{\frac{p}{p-1}}(0)-C_{1} t\right)^{\frac{p-1}{p}}
$$

with $C_{1}=c\left|x_{m}\right|^{-2} p(p-1)^{-\frac{p}{p-1}}$.

Therefore, from (32) we obtain that $F_{m}(t) \rightarrow 0$ as $t \rightarrow T^{*}=\frac{1}{C_{1}} F_{m}^{\frac{p}{p-1}}(0)$, that is $u(x, t)$ blows up in finite time.

As a result of these arguments, we have the following proposition:

Proposition 1. If $F_{m}(0)$ satisfies (30) for some $m \in \mathbf{N}$, that is

$$
F_{m}(0) \leq A\left|x_{m}\right|^{\frac{2(p-1)}{q-p-1}}
$$

with

$$
A=(p-1)^{-1}(2 c)^{\frac{1-p}{q-p-1}},
$$

then $u(x, t)$ blows up in finite time.

Here, we shall state the rest of the proof of Theorem (a) for $n \geq 2$.

Suppose that $u(x, t)$ be a nontrivial global solution. Thus, by Proposition 1, it follows that for any $m \in \mathbf{N}$

$$
F_{m}(0)>A\left|x_{m}\right|^{\frac{2(p-1)}{q-p-1}}
$$

that is

$$
\int_{B_{r, m}} u_{0}^{1-p}(x) \varphi_{m}(x) d x>(2 c)^{\frac{1-p}{q-p-1}}\left|x_{m}\right|^{\frac{2(p-1)}{q-p-1}} .
$$

Let $\varphi_{m}$ be radial function, that is $\varphi_{m}(x)=\varphi_{m}(\rho) \quad(\rho=|x|)$. Then, $\varphi_{m}(\rho)$ satisfies

$$
\left(\varphi_{m}\right)_{\rho \rho}+\frac{n-1}{\rho}\left(\varphi_{m}\right)_{\rho}+\lambda_{m} \varphi_{m}=0 \quad \text { in } B_{r, m} .
$$


Solving the equation (37), it follows that for some constant $a=a\left(\lambda_{m}\right)>0$

$$
\varphi_{m}(\rho)=a \rho^{-\frac{n-2}{2}} J_{\frac{n-2}{2}}\left(\sqrt{\lambda_{m}} \rho\right),
$$

where $J_{v}(z)$ is the Bessel function:

$$
J_{v}(z)=\left(\frac{z}{2}\right)^{v} \sum_{k=0}^{\infty} \frac{(-1)^{k}(z / 2)^{2 k}}{k ! \Gamma(k+v+1)}
$$

with the Gamma function

$$
\Gamma(k+v+1)=\int_{0}^{\infty} s^{k+v} e^{-s} d s .
$$

Then, we have

$$
\begin{gathered}
\varphi_{m}(\rho)=a \rho^{-\frac{n-2}{n}}\left(\frac{\sqrt{\lambda_{m}} \rho}{2}\right)^{\frac{n-2}{2}} \\
\times \sum_{k=0}^{\infty} \frac{(-1)^{k}}{k ! \Gamma\left(k+\frac{n-2}{2}+1\right)}\left(\frac{\sqrt{\lambda_{m}} \rho}{2}\right)^{2 k} \\
\leq a\left(\frac{\sqrt{c}}{2\left|x_{m}\right|}\right)^{\frac{n-2}{2}} \sum_{k=0}^{\infty} \frac{(-1)^{k}}{k ! \Gamma\left(k+\frac{n}{2}\right)}\left(\frac{\rho \sqrt{c}}{2\left|x_{m}\right|}\right)^{2 k}
\end{gathered}
$$

due to (21). Noting that $\rho \leq(1+r)\left|x_{m}\right|$ by (16), we obtain

$$
\begin{gathered}
\varphi_{m}(\rho) \leq a\left(\frac{\sqrt{c}}{2\left|x_{m}\right|}\right)^{\frac{n-2}{2}} \\
\times \sum_{k=0}^{\infty} \frac{(-1)^{k}}{k ! \Gamma\left(k+\frac{n}{2}\right)}\left\{\frac{(1+r) \sqrt{c}}{2}\right\}^{2 k} .
\end{gathered}
$$

Multiplying both sides of (36) by $\left|x_{m}\right|^{-n(p-1)}$, we have

$$
\begin{gathered}
\left|x_{m}\right|^{-n(p-1)} \int_{B_{r, m}} u_{0}^{1-p} \varphi_{m} d x \\
>(2 c)^{\frac{1-p}{q-p-1}}\left|x_{m}\right|^{\left(\frac{2}{q-p-1}-n\right)(p-1)} .
\end{gathered}
$$

Then, it follows from (42) that

$$
a\left(\frac{\sqrt{c}}{2}\right)^{\frac{n-2}{2}}\left|x_{m}\right|^{-\frac{n-2}{2}-n(p-1)} \sum_{k=0}^{\infty} \frac{(-1)^{k}}{k ! \Gamma\left(k+\frac{n}{2}\right)}
$$

$$
\begin{aligned}
& \times\left\{\frac{(1+r) \sqrt{c}}{2}\right\}^{2 k} \int_{B_{r, m}} u_{0}^{1-p}(x) d x \\
& >(2 c)^{\frac{1-p}{q-p-1}}\left|x_{m}\right|^{\left(\frac{2}{q-p-1}-n\right)(p-1)} .
\end{aligned}
$$

We note that

$$
-\frac{n-2}{2}-n(p-1)<0 \quad \text { if } \quad p>1 \text { and } n \geq 2,
$$

and

$$
\frac{2}{q-p-1}-n \geq 0 \quad \text { if } \quad p+1<q \leq p+1+\frac{2}{n} .
$$

Then, if $m \in \mathbf{N}$ is sufficiently large, the right-hand side of (44) is larger than the left-hand side of (44). Thus we arrive at a contradiction.

\subsection{Case II: $n=1$}

The inequality (44) lead to a contradiction for $p>\frac{3}{2}$ when $n=1$. So, we take the same strategy as in Li-Mu [11] and Pinsky [15] to prove Theorem for $n=1$ and $p>1$.

For $r>0$, let $\lambda_{r}>0$ denote the principal eigenvalue of $-\frac{\partial^{2}}{\partial x^{2}}$ with Dirichlet problem in $(-r, r)$, and let $\varphi_{r}(x)$ denote the corresponding eigenfunction, normalized by

$$
\int_{-r}^{r} \varphi_{r}(x) d x=1 .
$$

In fact,

$$
\varphi_{r}(x)=\frac{\pi}{4 r} \cos \frac{\pi x}{2 r} \text { and } \lambda_{r}=\frac{\pi^{2}}{4 r^{2}} .
$$

We define

$$
J_{r}(t)=\frac{1}{p-1} \int_{-r}^{r} u^{1-p}(x, t) \varphi_{r}(x) d x .
$$


Then we have

$$
\begin{gathered}
J_{r}^{\prime}(t)=-\int_{-r}^{r} \frac{u_{t}}{u^{p}} \varphi_{r}(x) d x \\
=-\int_{-r}^{r}\left(\frac{\partial^{2} u}{\partial x^{2}}+u^{q-p}\right) \varphi_{r}(x) d x .
\end{gathered}
$$

Integrating by parts, using (48), and noting that $\varphi_{r}^{\prime}(r)<0$ and $\varphi_{r}^{\prime}(-r)>0$, we obtain

$$
\begin{aligned}
& J_{r}^{\prime}(t) \leq \lambda_{r} \int_{-r}^{r} u \varphi_{r}(x) d x-\int_{-r}^{r} u^{q-p} \varphi_{r}(x) d x \\
& =\frac{\pi^{2}}{4 r^{2}} \int_{-r}^{r} u \varphi_{r}(x) d x-\int_{-r}^{r} u^{q-p} \varphi_{r}(x) d x .
\end{aligned}
$$

Since $p>1, q-p>1$, and $\int_{-r}^{r} \varphi_{r}(x) d x=1$, by Jensen' s and Hölder' s inequalities, we have

$$
\int_{-r}^{r} u^{1-p} \varphi_{r}(x) d x \geq\left(\int_{-r}^{r} u \varphi_{r}(x) d x\right)^{1-p}
$$

and

$$
\int_{-r}^{r} u^{q-p} \varphi_{r}(x) d x \geq\left(\int_{-r}^{r} u \varphi_{r}(x)\right)^{q-p}
$$

It follows from (52)-(53) that

$$
\begin{gathered}
\int_{-r}^{r} u^{q-p} \varphi_{r}(x) d x \\
\geq\left(\int_{-r}^{r} u \varphi_{r}(x) d x\right)^{q-p-1} \int_{-r}^{r} u \varphi_{r}(x) d x \\
\geq\left(\int_{-r}^{r} u^{1-p} \varphi_{r}(x) d x\right)^{-\frac{q-p-1}{p-1}} \int_{-r}^{r} u \varphi_{r}(x) d x \\
=\left[(p-1) J_{r}(t)\right]^{-(q-p-1) /(p-1)} \int_{-r}^{r} u \varphi_{r}(x) d x .
\end{gathered}
$$

Thus, by (51)-(54), we obtain

$$
\begin{gathered}
J_{r}^{\prime}(t) \leq \frac{\pi^{2}}{4 r^{2}} \int_{-r}^{r} u \varphi_{r}(x) d x \\
-\left[(p-1) J_{r}(t)\right]^{-\frac{q-p-1}{p-1}} \int_{-r}^{r} u \varphi_{r}(x) d x .
\end{gathered}
$$

Here, if $J_{r}(t)$ satisfies

$$
J_{r}(t) \leq \frac{1}{p-1}\left(\frac{2 r^{2}}{\pi^{2}}\right)^{(p-1) /(q-p-1)}
$$

for all $t \in\left[0, T^{*}\right)$, then by (55), it follows that

$$
J_{r}^{\prime}(t) \leq-\frac{\pi^{2}}{4 r^{2}} \int_{-r}^{r} u \varphi_{r}(x) d x .
$$

Hence, if (57) holds, then by (52)-(57), we have

$$
J_{r}^{\prime}(t) \leq-\frac{\pi^{2}}{4 r^{2}}(p-1)^{\frac{1}{1-p}} J_{r}^{\frac{1}{1-p}}(t)
$$

from which it follows that if $J_{r}(0)$ satisfies

$$
J_{r}(0) \leq \frac{1}{p-1}\left(\frac{2 r^{2}}{\pi^{2}}\right)^{\frac{p-1}{q-p-1}},
$$

then $J_{r}(t)$ decreases and

$$
J_{r}(t)<\frac{1}{p-1}\left(\frac{2 r^{2}}{\pi^{2}}\right)^{\frac{p-1}{q-p-1}} \text { for all } t \in\left[0, T^{*}\right]
$$

and an integration of (58) shows that

$$
J_{r}(t) \leq\left(J_{r}^{\frac{p}{p-1}}(0)-C_{2} t\right)^{\frac{p-1}{p}}
$$

with $C_{2}=\frac{\pi^{2}}{4} r^{-2} p(p-1)^{-\frac{p}{p-1}}$.

Therefore, from (61) we obtain that $J_{r}(t) \rightarrow 0$ as $t \rightarrow T^{*}=\frac{1}{C_{2}} J_{r}^{\frac{p}{p-1}}(0)$, that is $u(x, t)$ blows up in finite time.

As a result of these arguments, we have the following proposition:

Proposition 2. If $J_{r}(t)$ satisfies (59) for some $r>0$, that is

$$
J_{r}(0) \leq B r^{\frac{2(p-1)}{q-p-1}}
$$

with $B=(p-1)^{-1}\left(\frac{\pi^{2}}{2}\right)^{\frac{1-p}{q-p-1}}$, then $u(x, t)$ blows up in finite time.

Here, we shall state the rest of the proof of Theorem (a) for $n=1$.

Suppose that $u(x, t)$ be a nontrivial global solution. Thus, by Proposition 2, it follows that for any $r>0$ 


$$
J_{r}(0)>B r^{\frac{2(p-1)}{q-p-1}},
$$

that is

$$
\int_{-r}^{r} u_{0}^{1-p}(x) \varphi_{r}(x) d x>\left(\frac{\pi^{2}}{2}\right)^{\frac{1-p}{q-p-1}} r^{\frac{2(p-1)}{q-p-1}} .
$$

Note that

$$
\varphi_{r}(x)=\frac{\pi}{4 r} \cos \frac{\pi x}{2 r} \leq \frac{\pi}{4 r}
$$

by (48). Then, it follows that

$$
\frac{\pi}{4 r} \int_{-r}^{r} u_{0}^{1-p}(x) d x>\left(\frac{2}{\pi^{2}}\right)^{\frac{p-1}{q-p-1}} r^{\frac{2(p-1)}{q-p-1}} .
$$

Multiplying both sides of (66) by $r^{1-p}$, we have

$$
\frac{\pi}{4 r^{p}} \int_{-r}^{r} u_{0}^{1-p}(x) d x>\left(\frac{2}{\pi^{2}}\right)^{\frac{p-1}{q-p-1}} r^{\left(\frac{2}{q-p-1}-1\right)(p-1)} .
$$

We note that $p>1$, and

$$
\frac{2}{q-p-1}-1 \geq 0 \quad \text { if } \quad p+1<q \leq p+3
$$

Then, if $r>0$ is sufficiently large, the right-hand side of (67) is larger than the left-hand side of (67). Thus we arrive at a contradiction.

\section{Proof of Theorem (b)}

In this section, we shall prove the Theorem for the condition (b).

Since

$$
\liminf _{|x| \rightarrow \infty}|x|^{a} u_{0}(x)>0
$$

with

$0<a<\frac{2}{q-p-1}$, we have

$$
\begin{gathered}
\left|x_{m}\right|^{-\frac{2(p-1)}{q-p-1}} F_{m}(0) \\
=\frac{\left|x_{m}\right|^{-\frac{2(p-1)}{q-p-1}}}{p-1} \int_{B_{r, m}} u_{0}^{1-p}(x) \varphi_{m}(x) d x \\
\leq \frac{\left|x_{m}\right|^{-\frac{2(p-1)}{q-p-1}}}{p-1} \int_{B_{r, m}}|x|^{a(p-1)} \varphi_{m}(x) d x \\
\leq \frac{\left|x_{m}\right|^{-\frac{2(p-1)}{q-p-1}}}{p-1} \int_{B_{r, m}}\left\{(1+r)\left|x_{m}\right|\right\}^{a(p-1)} \varphi_{m}(x) d x \\
=\frac{(1+r)^{a(p-1)}}{p-1}\left|x_{m}\right|^{(p-1)\left(a-\frac{2}{q-p-1}\right)} \leq A
\end{gathered}
$$

with $A$ defined in (34) for $m \in \mathbf{N}$ large enough. Thus, $u(x, t)$ blows up in finite time by Proposition 1.

\section{References}

[1] H. Fujita, On the blowing up of solution of the Cauchy problem for $u_{t}=\Delta u+u^{\alpha+1}$, J. Fac. Sci. Univ. Tokyo 13 (1966) 109-124.

[2] V. A. Galaktionov, Blow-up for quasilinear heat equations with critical Fujita's exponent, Proc. Roy. Soc. Edinburgh 124A (1994) 517-525.

[3] V. A. Galaktionov, S. P. Kurdyumov, A. P. Mikhailov, A. A. Samarskii, Unbounded solutions of the Cauchy problem for the parabolic equation $u_{t}=\nabla\left(u^{\alpha} \nabla u\right)+u^{\beta}$, Soviet Phys. Dokl. 25 (1980) 458-459.

[4] Y. Giga, N. Umeda, Blow-up directions at space infinity for solutions of semilinear heat equations, Bol. Soc. Parana. Mat. 23 (2005) 9-28.

[5] J.-S. Guo, Y.-J. Guo, On a fast diffusion equation with source, Tohoku Math. J. 53 (2001) 571-579.

[6] K. Hayakawa, On nonexistence of global solution of some semilinear parabolic equation, Proc. Japan Acad. 49 (1973) 503-505.

[7] T. Igarashi, N. Umeda, Existence and nonexistence of global solutions in time for a reaction-diffusion system with inhomogeneous terms, Funkcialaj Ekvacioj 51 (2008) 17-37.

[8] T. Kawanago, Existence and behavior of solutions $u_{t}=\Delta\left(u^{m}\right)+u^{l}$, Adv. Math. 7 (1997) 367-400.

[9] K. Kobayashi, T. Sirao, H. Tanaka, On the blowing up problem for semilinear heat equations, J. Math. Soc. Japan 29 (1977) 407-424.

[10] T. Y. Lee, W. M. Ni, Global existence, large time behavior and life span on solution of a semilinear parabolic Cauchy problem, Trans. Amer. Math. Soc. 333 (1992) 365-378.

[11] Y.H. Li, C.L. Mu, Life span and a new critical exponent for a degenerate parabolic equation, J. Differ. Eqns. 207 (2004) 392-406.

[12] K. Mochizuki, K. Mukai, Existence and nonexistence of global solution to fast diffusions with source, Meth. Appl. 
Anal. 2 (1995) 92-102.

[13] K. Mochizuki, R. Suzuki, Critical exponent and critical blow-up for quasilinear parabolic equations, Israel $\mathrm{J}$. Math. 98 (1997) 141-156.

[14] K. Mukai, K. Mochizuki, Q. Huang, Large time behavior and life span for a quasilinear parabolic equation with slowly decaying initial values, Nonlinear Anal. 39 (2000) $33-45$.

[15] R. G. Pinsky, Existence and nonexistence of global solutions for $u_{t}=\Delta u+a(x) u^{p}$ in $\mathbf{R}^{n}$, J. Differ. Eqns. 133 (1997) 152-177.

[16] Y. W. Qi, On the equation $u_{t}=\Delta u^{\alpha}+u^{\beta}$, Proc. Roy. Soc. Edinburgh Sect. A 123 (1993) 373-390.

[17] M. Shimojō, On blow-up phenomenon at space infinity and its locality for semilinear heat equations (in Japanese), Master's Thesis, The University of Tokyo (2005).
[18] M. Shimojō, The global profile of blow-up at space infinity in semilinear heat equations, J. Math. Kyoto Univ. 48 (2008) 339-361.

[19] M. Shimojō, N. Umeda, Blow-up at space infinity for solutions of cooperative reaction-diffusion systems, Funkcialaj Ekvacioj 54 (2011) 315-334.

[20] F. B. Weissler, Existence and non-existence of global solutions for semilinear heat equation, Israel J. Math. 6 (1981) 29-40.

[21] M. Winkler, On the Cauchy problem for a degenerate parabolic equation, Z. Anal. Anwendungen 20 (2001) 677-690.

[22] M. Winkler, A critical exponent in a degenerate parabolic equation, Math. Meth. Appl. Sci. 25 (2002) 911-925.

[23] C. Yang, S. Zheng, S. Zhou, Critical exponents in a degenerate parabolic equation with weighted source, Applicable Analysis 92 (2013) 814-830. 